\title{
Enquête
}

Archives de la revue Enquête

$8 \mid 1993$

Varia

\section{Pourquoi Olympie}

\section{Paul Veyne}

\section{OpenEdition}

\section{Journals}

Édition électronique

URL : http://journals.openedition.org/enquete/168

DOI : 10.4000/enquete. 168

ISSN : 1953-809X

\section{Éditeur :}

Cercom, Éditions Parenthèses

\section{Édition imprimée}

Date de publication : 2 septembre 1993

Pagination : 3-25

\section{Référence électronique}

Paul Veyne, « Pourquoi Olympie », Enquête [En ligne], 8 | 1993, mis en ligne le 24 février 2006, consulté le 05 mai 2019. URL : http://journals.openedition.org/enquete/168 ; DOI : 10.4000/enquete.168 


\title{
Pourquoi Olympie
}

\author{
Paul Veyne
}

1 Lorsque nous approchons du village, mon chien Argos aboie d'une manière particulière, pour me signifier d'arrêter l'auto et de le laisser descendre : il veut faire le reste du trajet en courant derrière la voiture et en essayant d'aller plus vite que moi. Les animaux supérieurs savent jouer et concourir; le sport est donc encore plus «naturel » que la religion, puisque les humains n'ensevelissent leurs morts que depuis l'époque de Néandertal ${ }^{1}$. À vrai dire, la bonne question serait plutôt de savoir, non si les animaux sont joueurs, mais s'ils peuvent se comporter en spectateurs des jeux de leurs congénères. L'amour du sport et de la compétition est chose banale. Ce qui fait problème, en revanche, est que, dans la Grèce ancienne, durant plus d'un millénaire, le sport a eu une importance exceptionnelle, dont Olympie reste le symbole; plus encore, chez ce peuple intellectuel et artiste, il a eu une dignité sociale et culturelle exceptionnellement élevée. Nous allons nous demander pourquoi cette dignité. Cela ne consistera pas à découvrir les "causes " du phénomène olympique, mais plutôt à le rendre humainement plausible, à lui ôter son étrangeté, son apparence d'exception, en le rapprochant d'autres phénomènes qui ont avec lui une analogie qui, à première vue, n'apparaît pas; par exemple, un corroborée australien, la gloire nationale de Pouchkine en Russie ou les itinéraires consacrés du tourisme culturel ${ }^{2}$.

2 À Olympie, les jeux (ou, pour mieux dire, les compétitions ou agones) avaient lieu tous les quatre ans. Ils étaient organisés par les autorités d'une cité voisine, Elis, sur le territoire de laquelle se trouvait Olympie avec son bois sacré, son temple de Zeus et son stade; les revenus du sanctuaire permettaient à Olympie de financer la compétition. Tous les hommes libres, qu'ils fussent Grecs ou Barbares, étaient autorisés à assister aux épreuves, mais les femmes et les esclaves n'étaient pas admis; les concurrents, en revanche, devaient être grecs.

3 Les épreuves olympiques étaient exclusivement athlétiques : course de char, course à pied, boxe, lutte, etc. Mais il existait des centaines d'autres concours à travers le reste du monde grec, dont trois (à Delphes, à Némée et sur l'isthme de Corinthe) étaient presque aussi prestigieux qu'Olympie et attiraient des concurrents venus de toutes les cités grecques; ces autres concours comportaient souvent des épreuves « musicales » : chant et 
musique, théâtre ; la consécration d'un musicien était d'avoir été proclamé vainqueur à un des grands concours internationaux ; à chaque épreuve, il y avait (sauf match nul) un vainqueur et un seul; on était premier ou rien.

4 Musicaux ou athlétiques, ces concours ont passionné la société grecque, puis grécoromaine, jusqu'à triomphe du christianisme, et leur nombre n'a cessé de croître. L'antiquité a atteint son apogée économique sans doute au début du $\mathrm{III}^{\mathrm{e}}$ siècle de notre ère (la Tunisie, la Syrie et la Turquie connaissaient alors une prospérité qu'elles ne devaient retrouver que de nos jours); cette prospérité permet la multiplication des concours : chaque cité grecque, grande ou petite, veut avoir les siens. Olympie et les trois autres compétitions internationales demeurent les plus célèbres; seuls ils confèrent aux vainqueurs la célébrité. Mais Athènes, qui célébrait toujours ses Panathénées (comme au temps de la construction du Parthénon six cents ans plus tôt), suivait de près; même Sparte avait fini par avoir des jeux assez largement connus. Auguste avait fondé à Actium un cinquième concours grec international; Domitien fondera un concours grec, athlétique et musical, à Rome même, qui se déroulait au Capitole et sur le stade de la Piazza Navona (qui a conservé la forme de l'édifice). Au III siècle, Rome, capitale politique, capitale artistique aussi, fera concurrence à Olympie. Mais la moindre cité grecque du Moyen-Orient savait aussi attirer contre argent athlètes et musiciens.

5 Les grands concours donnaient lieu à un vaste rassemblement de peuple et pouvaient ainsi remplir plusieurs fonctions. Poètes et écrivains y donnaient des lectures publiques de leurs dernières productions (Hérodote lut ainsi son Histoire à Olympie) ; les orateurs y donnaient un échantillon de leur talent, les philosophes y prêchaient les dogmes de leur secte. Ce n'étaient pas là des compétitions : chacun profitait de la présence d'une grande foule. Le commerce en profitait aussi : les grands concours se doublaient d'une grande foire. Si bien, écrit un témoin ${ }^{3}$, que chacun suivait ses préférences: les uns se passionnaient à Olympie pour les épreuves athlétiques, les autres y venaient pour acheter et vendre, d'autres enfin venaient écouter orateurs et philosophes. Le public de la compétition olympique ne ressemblait guère à celui que réunit chez nous un match de football ou un championnat d'athlétisme. En outre, la célébrité internationale du concours faisait qu'on y venait moins par passion pour le sport que pour assister à un grand événement. Cicéron n'excluait pas l'idée d'aller un jour assister aux jeux d'olympie; on pense bien que ce n'était pas l'intérêt pour la boxe qui l'y poussait. Les jeux olympiques avaient pris une valeur culturelle, en ce sens qu'ils étaient considérés comme quelque chose d' « élevé », et non pas de corporel, de physique, de sportif.

Alors que l'intérêt pour le sport est «naturel », cette dignité culturelle constitue, à nos yeux de modernes, ce que l'olympisme a de surprenant et de spécifiquement grec. Les innombrables passages d'auteurs anciens qui parlent d'olympie le font avec une sorte de révérence; ils n'en parlent jamais comme d'une chose ludique ou snob. La victoire olympique sert proverbialement d'étalon de mesure ; « encore plus beau que d'avoir été plusieurs fois tout-puissant ou plusieurs fois vainqueur à Olympie », disait-on. La vie d'un héros, et d'un héros chanceux tel que Pélopidas, avait été "supérieure à toutes les victoires olympiques et pythiques»; un jour le roi Philippe de Macédoine reçut trois bonnes nouvelles à la fois: ses généraux venaient de remporter une victoire, son char avait gagné à Olympie et sa femme avait accouché d'un garçon, le futur Alexandre le Grand. Comme Epicure prétendait dédaigner les plaisirs de vanité, on lui rétorquait que lui-même serait mort de joie si on l'avait applaudi à Olympie. Lorsque les poètes ou les philosophes veulent faire une comparaison élevée, ils prennent olympie pour référence ${ }^{4}$. 
Pour faire comprendre combien la Sagesse est une grande chose, Diogène la dit supérieure à la victoire elle-même ${ }^{5}$. À notre époque, le terme de référence équivalent serait le Prix Nobel. De nos jours, une nation mesure sa vitalité et son prestige international au nombre de ses Prix Nobel. De même, lorsqu'Alcibiade voulut entraîner les Athéniens à la conquête de la Sicile, il leur expliqua qu'Athènes était puissante et qu'elle était encore plus redoutée, car lui-même, Alcibiade, venait de remporter plusieurs victoires à Olympie ; si bien que tous les Grecs « allaient jusqu'à s'exagérer » la puissance athénienne ${ }^{6}$.

7 Il existe une preuve a contrario de la dignité de l'olympisme: la contestation qu'en faisaient certains intellectuels. Ils ne le contestent pas à la manière d'un vieux professeur de chez nous qui stigmatiserait la passion du football, ils ne lui reprochent pas d'être un plaisir de bas étage et bon pour la plèbe: ils voient en lui un exemple des vaines occupations et des préjugés de tous les hommes; l'olympisme est la plus prestigieuse de leurs erreurs. «Il n'est pas juste », écrit Xénophane, « de mettre la vigueur corporelle audessus de la vraie sagesse ; un athlète qui sait boxer ou courir ne fera pas que la cité soit mieux gouvernée ». Xénophane se fait ici le porte-parole de la Sagesse, de même qu'un prêtre chrétien parle au nom de la vraie religion, et il critique une fausse opinion des gens bien éduqués, qui sont ses lecteurs et ses pairs. Il conteste la justification qu'on donnait à l'athlétisme, censé préparer aux vertus civiques. Dans l'antiquité, un philosophe était un personnage à part, une sorte de prêtre laïc, un profès de la sagesse ; sa mission est d'ôter à l'humanité les œillères qui la détournent de la Sagesse; de même les Pères de l'Église critiqueront l'arène, le Cirque et le théâtre, qui détournent les hommes de se vouer au seul souci de leur salut.

La critique de l'olympisme sera surtout le fait d'extrémistes, les philosophes cyniques, qui veulent ramener l'humanité à une simplicité naturelle et presque animale ; se passionner pour le sport est aussi ridicule que de s'enticher de culture, de confort, d'ambition ou d'amours; l'athlétisme est une vaine gloire ${ }^{7}$ : c'est la grande idée de Dion de Pruse. Et pourtant le même Dion, dans d'autres discours, fait un éloge transcendant d'un athlète indomptable, en qui il voit l'incarnation de la noble vertu de courage ${ }^{8}$. Noble courage : cet éloge du champion olympique était consacré chez beaucoup de penseurs; l'athlète était un héros de la «maîtrise de soi $^{9}$ ».

9 Car la pensée antique hésitait. D'un côté, l'olympisme est une des erreurs qui s'opposent à la vraie sagesse ; de l'autre, l'athlète est l'image vivante du sage, de cet homme courageux qui a triomphé de ses passions, qui ne tremble pas devant les menaces des puissants et qui ne craint pas la mort. L'athlète s'entraîne (askesai), comme fait le philosophe qui s'exerce à triompher de ses désirs ; l'entraînement d'un athlète exigeait de lui qu'il vécût comme un ascète: il croyait devoir s'abstenir des plaisirs de Bacchus et de Vénus, il devait résister à ces tentations. Or, dans l'antiquité, le courage est conçu comme une capacité de résistance plus que d'audace : il fait résister à la fatigue, à la souffrance, à la terreur, à la mort. L'athlète vainqueur que couronnent les juges de la compétition reçoit ainsi la récompense de sa vertu de résistance. C'est pourquoi il sera un modèle pour les chrétiens, comme il l'avait été pour les philosophes païens, dès les épîtres de saint Paul. En 203, la chrétienne Perpétua, qui va être martyrisée dans l'amphithéâtre de Carthage, a une vision dans sa prison: elle se voit en tenue d'athlète, de boxeur; elle triomphe de ses bourreaux et un ange, vêtu comme le président de la compétition, lui remet la palme du vainqueur, qui est devenue depuis ce que nous appelons la palme du martyre ${ }^{10}$. Dans les Oracles sibyllins, l'entrée des Élus au paradis est assimilée à l'entrée d'un athlète vainqueur 
qui, revenu dans sa cité natale, y est accueilli en triomphe, selon la coutume ${ }^{11}$. Le Christ a triomphé de la mort sur la croix, et c'est pourquoi, dans le Jugement dernier de MichelAnge, il est représenté sous l'aspect d'un athlète nu.

10 La dignité de l'athlétisme antique n'avait donc rien d'esthétique, malgré Winckelmann: c'était une dignité éthique, qui valait bien la dignité artistique des compétiteurs qui affrontaient les épreuves musicales; l'athlète est, dans son genre, au moins l'égal de ces virtuoses. Chaque génération, dit Plutarque, «se passionne pour les artistes, les comédiens et les athlètes de son époque, et méprise ceux de la génération précédente ${ }^{12}$ ». Toutefois, on peut penser que la morale doit prévaloir sur la beauté : un athlète athénien se loue de l'avoir emporté, «non dans une épreuve de chant, mais bien de vertu ${ }^{13}$ ». Le prestige de l'athlète est plus grand que celui d'un musicien vainqueur, écrit Philostrate, puisque Olympie, où il n'y a pas d'épreuves musicales, est le plus grand des grands concours $^{14}$; ce qui donne beaucoup à penser ; nous pourrions être tentés de supposer que les Grecs ont prêté aux athlètes les plus hautes vertus, au nom d'une certaine " conception » qu'ils se faisaient du type humain de l'athlète. Ne serait-ce pas plutôt l'inverse? Ils n'admiraient les athlètes que par voie de conséquence: comme la compétition olympique était culturellement élevée à leurs yeux, il leur a fallu, au nom de la logique, reverser sur les compétiteurs une partie de cette élévation, afin de justifier le prestige d'olympie.

11 Ils pouvaient le faire d'autant plus facilement que les athlètes appartenaient généralement à la bonne société possédante qui vivait de loisir et qui avait du temps et des rentes pour s'entraîner ${ }^{15}$. On avait plus d'estime sociale, me semble-t-il, pour un athlète que pour un sculpteur ou un grammairien, pauvres hères qui vivent de leur travail ${ }^{16}$ (ce qui n'empêchait pas d'admirer les chefs-d'œuvre de la sculpture et de placer très haut l'érudition littéraire). Parlons argent. Il existait trois genres de compétitions ; les quatre grands concours internationaux (celui d'olympie en premier lieu) décernaient aux vainqueurs des différentes épreuves une couronne et pas de monnaie; d'autres concours rapportaient couronne et argent ; d'innombrables concours locaux rapportaient de l'argent seulement : sans oser décerner de glorieuse couronne, ils attiraient par l'appât monétaire des athlètes dans l'obscure petite cité qui organisait la compétition. Ce qui rapportait le plus était, paradoxalement, une victoire dans les quatre grands concours: Olympie ne versait pas d'argent, mais, en revanche, la cité grande ou petite qui était la patrie du vainqueur était légalement tenue de lui décerner une récompense monétaire, une rente, des exemptions d'impôts et de grands honneurs. Si bien que les vainqueurs olympiques étaient, en dernière analyse, les mieux rétribués.

Amateurisme ou professionnalisme? Les athlètes vivaient-ils du sport et avaient-ils besoin du sport pour vivre? Dans les conditions économiques de l'antiquité, la question n'a guère de sens; mieux valait être un rentier pour commencer une carrière, nous l'avons dit, mais cette carrière enrichissait encore davantage un champion. Nous dirions que les athlètes étaient des professionnels, en cela qu'ils s'adonnaient exclusivement à leur entraînement et aux compétitions, qui les faisaient voyager dans tout le monde grec, puis gréco-romain, jusqu'à Rome, Carthage ou Vienne (dans la vallée du Rhône); professionnels aussi, en cela que le sport leur rapporte de l'argent. Mais amateurs, en cela qu'ils n'ont pas besoin de travailler pour vivre et qu'ils appartiennent en majorité à la leisure class ; amateurs, enfin, en cela que l'athlétisme était considéré comme une activité élevée, dont on faisait profession (Beruf), qui était une vocation (Berufung); un athlète était le profès d'une "profession libérale ", à la manière d'un philosophe, d'un rhéteur, 
d'un poète ou d'un médecin. Lorsque le champion est le rejeton d'une noble famille, il ne la déshonore pas en se consacrant au sport: des boxeurs sont issus de sénateurs romains et même de consuls ${ }^{17}$; s'il est d'origine plébéienne, ses victoires le feront entrer dans la classe gouvernante de sa cité.

Nobles amateurs ou vils professionnels ? Ce faux problème a été longtemps discuté par les historiens, parce qu'il permettait de résoudre, grâce à la notion de "décadence", l'énigme qu'était la dignité antique de l'olympisme ${ }^{18}$; de même, la décadence des beauxarts était attribuée à l'appât du gain, qui avait supplanté l'antique amour de la gloire ${ }^{19}$. En fait, l'athlétisme antique a toujours été plus aristocratique que plébéien. mystérieux de l'athlétisme en Grèce : à l'époque hellénistique (tenue gratuitement pour décadente, parce que non "classique » ni "primitive»), la popularité du sport était attribuée à l'appât du gain et à l'abaissement des esprits ; à l'époque archaïque, la même popularité était rapportée romantiquement à une antique simplicité, parfumée d'aristocratisme primitif ; c'est la Grèce de Hôlderlin ou de Heidegger ${ }^{20}$, ce printemps d'un monde archaïque et pur. Nudité des athlètes, sens de la beauté, vaillance, goût de la gloire.

La singularité de l'olympisme sera donc expliquée par le moment historique (primitif ou décadent), par le milieu (aristocratie) ou par la race («dorienne» ou, plus largement, hellénique). Le goût du sport sera tenu pour une singularité nationale qu'il ne reste plus qu'à admettre : les Grecs étaient faits ainsi, voilà tout. Ils n'en sont que plus admirables, puisqu'au fond ce goût a une saveur primitive et noble: des aristocrates aiment à se mesurer entre eux et à primer. On aboutit ainsi à une théorie longtemps consacrée : le prestige des compétitions (ou agones) était dû au « sens agonistique » de la vieille Grèce; pour que ce goût soit une singularité ethnique et que l'explication ne soit pas une vulgaire tautologie, il faut que les autres peuples aient été dépourvus de ce sens agonistique et du moindre goût pour le sport; les peuples de l'Orient ancien, a-t-on cru longtemps, n'étaient guère sportifs. Nous savons aujourd'hui que c'est là une légende : le sport était apprécié dans les hautes cultures anciennes, Orient et Égypte compris ${ }^{21}$. Nous avons déjà vu que la singularité grecque n'a pas consisté à aimer le sport, mais à lui conférer cette « dignité culturelle » dont nous reparlerons.

On ne peut guère parler d'un sens hellénique de la rivalité en général (une guerre entre deux cités, la carrière politique d'un ambitieux et une épreuve de course à pied n'ont que peu de rapport); en revanche, il est légitime de parler d'un sens grec de la forme institutionnelle qu'est la compétition ; les Hellènes raffolaient des concours et en créaient à chaque occasion. Il y avait à Tarente des concours de broderie, à Pergame des compétitions de médecine, sans oublier les concours de beauté, qui ne sont pas une légende. Le système éducatif s'accompagnait de compétitions. Les enfants grecs de naissance libre allaient à l'école, où ils apprenaient à lire, à écrire, à chanter, à déchiffrer Homère ; au gymnase, ils écoutaient souvent des conférences de rhétorique et parfois de philosophie, qui servaient d'interlude à leurs séances d'athlétisme. Et, à la fin de chaque année, chaque école célébrait sa compétition sportive et musicale, réservée à ses élèves. Ce n'était pas là un " examen de fin d'année ", destiné à trier les élèves qui passeraient dans la "classe supérieure ", comme on l'a cru parfois (car, en ce cas, un seul élève, le vainqueur, serait passé, puisqu'il n'y avait qu'un seul vainqueur à chaque compétition : on ne montait pas à trois sur le podium...). Le concours permettait plutôt de juger de la qualité des maîtres de musique et de gymnastique, de saluer de jeunes vedettes et 
d'apprécier la bonne éducation que la cité donnait à ses enfants. Chaque cité était jugée physionomiquement par les étrangers sur la qualité éthique et esthétique de ses concours.

17 Les concerts publics eux-mêmes avaient la forme de compétitions. Un jour, à Naples, qui était alors une cité grecque, Sénèque passa devant le petit théâtre (dont les ruines sont encore visibles), et voici ce qu'il raconte ${ }^{22}$ : «Cet odéon était bondé : on y discutait passionnément pour décerner le prix du meilleur flûtiste; un trompettiste de l'école grecque et de la latine avaient aussi leurs partisans »; Naples était un centre culturel de réputation internationale, et plusieurs virtuoses se produisaient ce jour-là dans ce qui serait pour nous un concert.

Plusieurs particularités de la civilisation antique ont, sinon provoqué, du moins rendu possible la valorisation des compétitions athlétiques. D'abord, en matière de sport, on ne distinguait pas entre une culture populaire et une culture noble; on ne distinguait pas non plus entre le travail et le loisir. On ne distinguait guère non plus entre les fonctions « sérieuses » de l'État (sauver la collectivité, etc.) et les coutumes de ce que nous appelons la société civile.

19 Chez nous, on fait une distinction entre les sports de la bonne société (le polo et, récemment encore, le tennis) et les sports populaires (le football); les compétitions grecques, en revanche, étaient l'affaire de tous les citoyens sans distinction. On ne les opposait pas davantage à la culture élevée (littéraire et musicale) : athlétisme et musique appartenaient également aux coutumes de l'aristocratie. On sait combien les valorisations sociales sont traditionnelles; la dignité du sport en Grèce est due à un fait de chronologie relative, c'est-à-dire à un hasard historique : culture littéraire et sport remontent à une même antiquité ; Achille jouait de la musique, organisait une compétition athlétique pour célébrer les funérailles de Patrocle, et écoutait des aèdes qui lui récitaient des chants épiques. Chez les modernes, en revanche, les sports ne font leur véritable apparition qu'au XIX ${ }^{e}$ siècle, tandis qu'en Italie, dès le XVI ${ }^{e}$ siècle, et en France depuis le XVII ${ }^{e}$ siècle, on parle littérature dans les salons de la noblesse; à cette époque, la bonne société s'intéressait à ses bals et à la chasse, non à l'athlétisme. Durant le XIX ${ }^{e}$ siècle bourgeois, le lycée et le Gymnasium enseigneront le latin (et, en Allemagne, le grec), mais non les sports.

Par ailleurs, un frein n'existait pas : le sérieux du travail, le puritanisme bourgeois dont parle Max Weber ; une aristocratie ne qualifie pas de travail ses activités, et le travail du peuple n'est pas une dignité ; les plaisirs et spectacles ne s'opposent pas à une partie sérieuse de la vie privée. Il suffit de songer, de nos jours, à la passion du football dans les foules du sud de l'Europe, de l'Amérique du Sud, et dans les villes de l'Angleterre qui sont sinistrées par le chômage; ou de se souvenir d'un mot de Nietzsche: les bourgeois ne savent pas que faire de la religion, parce qu'elle n'est ni un travail, ni un plaisir.

21 Enfin, une cité grecque n'est pas une abstraction, une institution chargée de remplir les fonctions de la Raison d'État ; c'est une collectivité concrète qui est réputée se gouverner elle-même; comme un individu vivant, elle choisit ses mœurs et coutumes; c'est pour cela qu'elle établit ses fêtes religieuses publiques et ses compétitions (et non pas parce que les gouvernants voudraient témoigner aux gouvernés qu'ils ne dédaignent pas leurs plaisirs). Société civile et État sont mal séparés ; certes, le plus souvent, la cité laisse ses membres décider de leur religiosité personnelle; mais elle peut toujours reprendre l'exercice de son droit de contrôle de tous sur tous : Socrate en fit la fâcheuse expérience, 
qui fut condamné à mort pour avoir cru en d'autres dieux que ceux de sa cité. La frontière entre les lois et les simples coutumes n'était pas étanche; les fêtes et les compétitions pouvaient donc être une affaire d'État. Elles le redeviennent au $\mathrm{xx}^{\mathrm{e}}$ siècle, mais pour d'autres raisons : l'idéal du Welfare State fait sortir l'État de ses fonctions étroitement politiques, pour lui faire embrasser tous les intérêts de la population qu'il gouverne ; et nations ou partis utilisent nos Jeux Olympiques comme tribune où montrer au monde entier leur existence ou leurs revendications.

Nous, modernes, avons, comme on voit, des raisons sérieuses de nous intéresser à l'olympisme. Et les Grecs? N'auraient-ils pas eu des raisons non moins « sérieuses », eux aussi ? Ne fallait-il pas qu'elles soient sérieuses, pour être à l'échelle du phénomène et pour expliquer l'importance qu'avaient l'athlétisme et Olympie aux yeux des Grecs? On a donc supposé que la passion de l'athlétisme avait des raisons importantes et relatives à de grands intérêts : former des citoyens et des guerriers, renforcer l'identité nationale ${ }^{23}$, honorer les dieux. Civisme, nationalisme, religion. L'explication, comme on voit, est fonctionnelle et rationnelle; elle dit à quoi servait l'olympisme, qu'elle considère comme un moyen approprié à des fins. Malheureusement, on va voir qu'aucune de ces trois explications n'est correcte: l'olympisme ne servait à rien, ou plutôt la sociologie fonctionnelle n'est qu'une rationalisation ${ }^{24}$. La bonne explication sera moins rationnelle; elle ne se rapportera pas à de grands intérêts, mais mêlera les hasards des phénomènes de mode et une tendance fréquente à donner à la culture des formes canoniques et, pour ainsi dire, des classiques.

L'explication par l'éducation civique était celle des Grecs eux-mêmes. Dans un dialogue amusant, l'Anacharsis, le sceptique Lucien met face à face un sage barbare, Anacharsis, et un Sage athénien, Solon. Le Barbare commence par s'étonner du spectacle des gymnases grecs : on y voit toute la jeunesse d'une cité s'adonner à la gymnastique, à la lutte, à la boxe; Solon réplique que les usages d'un peuple paraissent toujours extravagants aux autres nations ; que les exercices physiques sont motivés par le goût de la gloire et que cette gloire est légitime, puisque l'athlétisme forme des citoyens tempérants et des guerriers courageux. Anacharsis n'en croit pas un mot : il aperçoit la disproportion entre la grandeur de cette préparation et la minceur des résultats; pour lui, ces exercices sont inutiles et sont le fruit de la mode, de l'oisiveté et de la vanité ; ce qui est aussi l'opinion de Lucien, qui est secrètement agacé par l'athlétisme, car toutes les superstitions l'irritent; l'olympisme, à ses yeux, n'est qu'une superstition grecque. On songe à l'agacement que donne à certains intellectuels russes le culte exclusif de Pouchkine, ou aux estampes où Hokusai raille l'idolâtrie que ses compatriotes vouent au paysage du Mont Fuji.

24 Lucien n'a pas tort. Les gymnases avaient perdu depuis longtemps leur rôle de terrains de préparation militaire; ils n'éduquaient pas les adolescents pour la vie civique, mais selon un idéal humain plus général ; leur enseignement était gymnique, littéraire et musical. Il en allait de même de l'éphébie, où les jeunes hommes recevaient une formation sportive et intellectuelle; on a parlé d'une Institution für die Söhne der upper class, comparable aux public schools britanniques et aux colleges les plus huppés des États-Unis ${ }^{25}$.

D’une façon générale, il est plutôt rare qu'un système éducatif soit réellement fonctionnel et qu'il prépare les enfants à leur métier futur de citoyen ou de travailleur ; plus souvent, les adultes projettent sur les chères têtes juvéniles l'idéal humain et culturel de leur époque. Notre enseignement a reposé sur le latin, clé d'une culture conçue comme tradition; à Rome, il reposait sur l'étude de la rhétorique, cette fiction d'une synthèse 
entre parole, raison, persuasion et pouvoir. L'importance que l'éducation grecque attachait à l'athlétisme confirme que l'olympisme faisait partie de la culture d'un gentleman.

Nous venons de parler d'un idéal qui fut celui des Grecs et de la culture hellénique. Souvent une culture a pour frontières celles d'un peuple. On voit alors comment la plume d'un historien peut facilement déraper et glisser vers un mot à la mode, celui d'identité nationale. Ce mot est dangereusement équivoque. Il est parfois neutre et n'est qu'une manière compliquée de constater, par exemple, que les compétitions étaient une des composantes de la civilisation grecque. Mais, plus souvent, le mot est sournoisement chargé de pathos: il insinue qu'une identité est toujours menacée ou inquiète, que les peuples doutent de leur identité et la chérissent et qu'ils éprouvent le besoin de l'affirmer à la face du monde. Dès lors, nous nous flatterons d'avoir trouvé une explication sérieuse et fonctionnelle à l'olympisme : les Grecs allaient en foule à Olympie parce qu'ils étaient tourmentés du besoin d'affirmer leur identité à leurs propres yeux et face aux Barbares ou aux Romains.

Je crains que ce soit pour notre propre satisfaction intellectuelle que nous prêtons aux Grecs ce mobile. Qui nous a dit que leur identité les tourmentait à Olympie? Ils étaient peut-être paisiblement imbus de leur supériorité; ils pouvaient aussi savourer une de leurs fêtes nationales, non parce qu'elle était nationale, mais parce que c'était une fête ; peut-être aussi allaient-ils nombreux à Olympie parce qu'en effet le rayonnement de cette compétition s'étendait à la grécité tout entière ; c'est-à-dire qu'ils y allaient par curiosité et snobisme.

Cette dernière raison est la bonne. Les Grecs allaient nombreux à Olympie pour la simple raison qui pousse de nos jours une foule de personnes fortunées à aller se presser à nos Jeux Olympiques: parce que l'une et l'autre compétition est et était un événement « mondial ». Loin de symboliser une identité inquiète, Olympie se sentait « œcunémique ${ }^{26}$ ", comme on disait alors. La fête était "pan-hellénique ", concurrents et spectateurs y venaient de loin ; les juges des épreuves étaient appelés « juge des Grecs» en général, de quelque cité grecque que les athlètes fussent citoyens ; le vainqueur se haussait d'un coup au niveau international ; chaque fois qu'on parlait d'Olympie, les mots : «tous les Grecs » revenaient dans les propos et remplissaient la bouche.

Olympie se sentait aussi pacifique qu'œcuménique. La compétition ne pouvait servir à des réalités de patriotisme de clocher (Lokalpatriotismus) entre cités, car les cités grecques étaient beaucoup trop nombreuses et dépassaient le millier ; le vainqueur pouvait n'avoir qu'un ou deux compatriotes parmi les spectateurs. Olympie suscitait le rêve que nos propres Jeux étendent à toute la planète, celui d'une humanité pacifiée et unie, où triomphait le seul mérite, sous les yeux de tous.

On ne nous relate pas d'incidents qui auraient utilisé la compétition comme support de revendications nationales ou sociales. On apercevait avec fierté, parmi les spectateurs, des rois étrangers ou des sénateurs romains ${ }^{27}$; parmi les concurrents, des natifs de cités nouvellement hellénisées ${ }^{28}$, ou quelque prince impérial qui prend part, par cocher et chevaux interposés, à une course de chars qu'on a ressuscitée en son honneur ${ }^{29}$. Avoir vu tout cela de ses yeux était flatteur; l'information n'est pas toujours un «capital symbolique » (souvent elle ne sert à rien), mais elle demeure un privilège. Lucien, ce détracteur d'Olympie, ne se prévaut pas moins d'y être allé six fois ${ }^{30}$. 
31 Loin d'être inquiets de leur identité, les Grecs avaient la certitude de leur supériorité (dans l'empire romain, qu'on pourrait appeler aussi bien gréco-romain, c'était l'autre peuple, les Romains, qui avaient un complexe d'infériorité). Il y a une nuance particulière dans leur nationalisme: ils parlent de l'olympisme avec une ferveur et une tendresse sensibles. On songe à une définition de l'Encyclopodia Britannica, édition 1910: "Cricket: the national summer pastime of the English race ». Les Grecs se complaisent pareillement dans les institutions et coutumes qui sont les leurs, y retrouvent leur propre physionomie, les chérissent. En tête de la liste canonique des joyaux nationaux venaient olympie et Homère. Nous reviendrons plus loin sur cette définition extrinsèque de la culture, où entrent une complaisance à soi-même et une volonté de fidélité à soi-même.

Les compétitions avaient par ailleurs un caractère qui semble, à première vue, étranger aux Jeux modernes : c'étaient des fêtes religieuses. À Olympie, la compétition se déroulait dans un sanctuaire de Zeus, à côté d'un temple du dieu et d'un bois sacré ; le premier jour et la matinée du troisième étaient réservés à des processions, des sacrifices, une hécatombe; le déroulement des épreuves s'accompagnait de gestes pieux; chaque vainqueur offrait ainsi à Zeus un sacrifice de remerciement et lui consacrait sa couronne d'olivier. Il était difficile de tirer au clair cette juxtaposition de sacré et de profane ; les Anciens ne savaient pas trop si les statues de vainqueurs qui se dressaient dans le sanctuaire devaient être considérées comme des ex-voto aux dieux ou des monuments à la mémoire de l'athlète. On a vu aussi qu'une foire se tenait pendant la compétition et qu'on y trouvait toutes les réjouissances, culturelles et autres, qui accompagnent d'ordinaire un grand rassemblement de peuple.

La sécularisation du monde moderne nous porte par réaction à supposer que les peuples anciens étaient toute piété. En réalité, il faut, en pareille matière, distinguer des cas d'espèce et raisonner empiriquement : quels étaient les sentiments des spectateurs ? Les innombrables témoignages antiques ne laissent ici aucun doute : c'étaient des amateurs de sport ou des curieux, qui n'étaient pas venus par dévotion; pas plus que nous n'allons à nos Jeux Olympiques par piété envers les idéaux de Coubertin ou pour la seule cérémonie de la Flamme Olympique.

Le lecteur nous rétorquera peut-être, à juste raison, que la juxtaposition du sacré et du profane n'était pas choquante dans le paganisme, qu'elle ne gênait nullement un authentique sentiment de piété et qu'il ne faut pas juger ce mélange avec la rigueur de l'esprit protestant. Dans la Grèce ancienne, les foires ou "panégyries » se tenaient à côté des grands sanctuaires, le même jour que le grand sacrifice annuel; il en va de même dans la Grèce d'aujourd'hui ${ }^{31}$ : le jour du pèlerinage, on voit se dresser à côté de l'église des stands de fête foraine, un marchand de brochettes de viande ou souvlàkia et une piste de danse ; la grande foire n'est pas loin, avec étalages de blue-jeans, d'icônes fabriquées à Hongkong et de bibelots africains.

Deux cas sont cependant à distinguer, et les Grecs eux-mêmes faisaient la distinction : les solennités et sacrifices consacrés à un dieu, les compétitions célébrées " en l'honneur » d'un dieu ou d'un défunt; le concours olympique se déroulait ainsi « en l'honneur » de Zeus. Cette distinction correspondait bien aux sentiments des spectateurs; quand il y avait juxtaposition d'un sacrifice et d'une foire, chaque pèlerin y trouvait ce qu'il voulait : de la piété, des plaisirs ou les deux à la fois ; le cas des compétitions était différent : les spectateurs n'avaient pas fait le voyage d'olympie pour y honorer Zeus, mais pour assister aux épreuves. La compétition « en l'honneur » de Zeus ne juxtaposait pas le sacré et le profane : elle était une solennisation religieuse du profane. Chez nous, la cérémonie 
de la Flamme Olympique est une autre forme de solennisation. La solennisation du sport n'était nullement le résultat d'une dégradation, d'une désacralisation: dès l'époque homérique, il en allait de même. À la fin de l'lliade, Achille solennise les funérailles de Patrocle en instaurant une compétition athlétique; or, pendant ces jeux funèbres en l'honneur du défunt, les concurrents, les spectateurs et le poète lui-même ne s'intéressent plus qu'aux épreuves, et Patrocle est momentanément oublié.

L'utilisation du sacré comme simple moyen de solenniser ne doit nullement rendre suspecte la sincérité religieuse des Grecs, tout au contraire ; le sacré ne se réduisait pas pour eux à un prétexte : il faut croire aux dieux pour solenniser ainsi. Ce qui le prouve $a$ contrario est que nous-mêmes avons recouru à un mode de solennisation autre que la religion pour nos propres Jeux Olympiques. Quelqu'un qui ne croirait pas en la musique n'aurait pas imaginé de rehausser les cérémonies européennes en y faisant exécuter l' Hymne à la Joie. On retrouve ici un problème qui reparaît partout sous différentes formes : un sentiment donné, la piété par exemple, existe-t-il par lui-même ou n'est-il que la résultante d'un contenu? Le sacré n'était-il qu'une sorte de "couverture idéologique ", fabriquée, pour les besoins de la cause, par le désir de solenniser? Ou bien n'avait-on, pour cela, qu'à puiser dans un sens naturel du sacré ? Pour Marx, le contenu de l'intérêt de classe donne naissance à l'idéologie, et la misère suscite l'opium du peuple ou la littérature d'évasion; pour Freud, le contenu refoulé du désir fait naître le symbolisme. Peut-on espérer que les enfants jouent aux gangsters ou se délectent de films de violence à la télévision pour défouler leurs pulsions sadiques? Si c'était vrai, il faudrait croire que ces films opèrent une katharsis et purgent les bambins de leurs vilaines pulsions. Si, au contraire, l'imaginaire existe par lui-même et ne se réduit pas à ce que l'abréaction le fait être, alors les films de violence pourront aussi bien renforcer les pulsions sadiques des uns que purger celles des autres ${ }^{32}$.

Que ce soit à Olympie ou dans n'importe quelle cité, le sport grec gravite autour de la compétition. Loin d'en être la cause, la religion est une simple conséquence du succès de ce "phénomène de mode ", qu'elle s'est bornée à solenniser. Ce qui est moins banal est que l'olympisme a été élevé au rang d'un « classique » (ce qui a entraîné l'idéalisation du type éthique de l'athlète). D'où vient ce succès d'Olympie? Il est dû à des hasards historiques dont nous ignorons le détail ${ }^{33}$, mais on en devine le mécanisme: le succès attire le succès. Si le succès initial atteint un point critique et que le nombre des convertis devient assez élevé, la foule des indifférents se convertira à son tour; non par instinct d'imitation, mais parce que la nouvelle mode leur semble être désormais la manière normale de se comporter. Voilà cette mode élevée au rang de tradition. À l'époque hellénistique et impériale, cités, mécènes, rois et empereurs fondent des compétitions, des agones athlétiques ou musicaux, dès qu'ils veulent s'élever au-dessus de la basse région de la Machtpolitik et des intérêts matériels, et se faire voir dans le beau rôle de héros culturels. Les Grecs sont devenus le peuple «agonistique » dont parle Burkhardt parce que les agones ont eu du succès chez eux; les agones n'ont pas eu de succès parce que les Grecs étaient agonistiques. Ce qui passionne les différents peuples n'est pas toujours ce que nous-mêmes considérerions comme socialement ou humainement important.

38 L'olympisme était important parce qu'il était considéré comme élevé, ce qui n'est pas le cas du sport chez nous: nos Jeux Olympiques ne sont qu'un "phénomène de masse ", auquel les États modernes attachent de l'importance à cause de leurs effets sur l'opinion des foules et parce qu'un welfare State veille à satisfaire l'ensemble de sa population. 
L'olympisme, lui, faisait partie de la culture, au sens le plus élevé de ce mot. Que veut dire "culture », en effet? Au moins trois choses différentes, que le sociologue wébérien J.C. Passeron a finement distinguées ${ }^{34}$.

En un premier sens du mot, la culture est ce qu'un observateur étranger peut décrire comme manières de faire et de penser qui sont habituelles chez un peuple déterminé ; la culture grecque était agonistique, la culture japonaise actuelle s'occidentalise de plus en plus... En un autre sens, qui est différent et trompeur, la culture est ce que le peuple considéré dit de lui-même, ce qu'il croit être (souvent à tort), ce par quoi il veut se définir, ce qu'il ordonne à ses propres membres de faire ou du moins de professer. Le mot a enfin une troisième signification qui est la plus élevée: une culture est un canon d'œuvres ou de pratiques qui sont hautement valorisées et tenues pour classiques. « Si l'on me demandait quel est le meilleur des quatre éléments ", écrivait à peu près le poète Pindare, « je répondrais que c'est l'eau ; la matière la plus précieuse ? Je dirais : l'or ; et, si on m'interroge sur les compétitions, je n'hésiterai pas : la plus glorieuse est Olympie ${ }^{35}$. »

Les choses qui sont culturelles en ce troisième sens sont intenses et durables, et non banales et éphémères. Chez nous, ce sont surtout certains livres, certains tableaux, certaines partitions; mais, en des époques plus anciennes, ce pouvait être aussi des pratiques : l'olympisme non moins qu'Homère; la culture était ce par quoi un peuple se montrait sous son meilleur jour ou ce par quoi une aristocratie se distinguait : manières mondaines ou chevaleresques, solennités, bals, corroborée, tournois.

Ce qui a permis à l'olympisme d'appartenir à la culture élevée est que le sport est un plaisir et qu'il relève du sublime. Le sport n'a pas l'âpreté des activités politiques et économiques; il n'est pas dissymétrique comme la politique : le champion n'est pas un chef et les spectateurs sont ses juges. Une compétition est une activité terminale et complète, dont on sort satisfait ; à la différence de planter ou semer, ce n'est pas une étape dans un long processus; c'est une satisfaction individuelle, alors qu'une victoire appartient à toute l'armée qui l'a remportée; comme la nourriture, c'est une satisfaction immédiate, et non pas différée comme celle des activités acquisitives. On peut en dire autant de la lecture, de la musique, de toutes les pratiques de l'homo ludens. Comme les chefs-d'œuvre, les records sont des exploits; comme les artistes, les champions font vibrer en nous le sentiment du sublime.

L'olympisme était donc un classique. Une culture se compose ainsi de choses qu'il « faut avoir lues ", vues, entendues ou faites ; quand le tourisme culturel cesse d'être ruineux et devient usuel, il se fige en itinéraires canoniques, du Duomo à la Signoria, devant la Joconde ou Néfertiti. Il y a les quatre grandes compétitions, les dix grands orateurs, les quatre sectes philosophiques et les sept merveilles du monde. Le cas d'Homère est caractéristique: c'était plus qu'un chef-d'œuvre et ce n'était cependant pas un livre saint; Homère n'était pas le plus grand poète grec, il était autre chose qu'un auteur : une autorité, une superstition ; il serait pourtant très creux de répéter que, chez Homère, les Grecs voyaient représentées toutes leurs valeurs; on dit cela faute de mieux, faute de pouvoir expliquer cet étrange prestige. L'œuvre d'Homère est aussi particulière que toute autre œuvre d'art et elle ne résume pas plus les valeurs grecques que Pouchkine, cette autre idole, ne résume celles des Russes. Le fait n'en est pas moins là ; il fait voir qu'une culture peut se fixer en un canon, au sens où l'on parle du canon de l'Écriture sainte. Aristote écrivait que les Crétois de son temps avaient presque cessé d'être des Grecs, si ce n'était qu'ils connaissaient encore Homère. 
La fixation d'un canon culturel est une manière de maîtriser la culture, de même que la symétrie est la manière archaïque ou naïve de mettre de l'ordre dans le chaos du visible et de maîtriser la peinture. Elle aboutit à donner de la culture une définition extrinsèque, à l'organiser selon un souci qui n'est pas culturel, celui de mériter le beau nom de Grec ou le titre de personne cultivée, au lieu de se mouvoir librement et sans souci à travers le visible ou parmi les livres, ou de décréter que les sept merveilles du monde ne valent pas le Parthénon (qui ne figure pas dans la liste). Ce qui met fin à la tragédie de la culture dont parle Simmel ${ }^{36}$, à l'oscillation entre deux pôles qui compose toute notre existence : individualisme ou recherche d'un consensus, volonté d'intensité ou quête de la sécurité, ouverture ou clôture, auto-affirmation ou légitimité, en concluant chaque fois en faveur du second pôle. La canonisation de l'olympisme a ratifié les décrets de la mode et n'a pas répondu à un besoin d'identité ni à une fonction éducative, etc. : elle a été un épisode de la tragédie de la culture. Une des issues de cette tragédie est de considérer une chose particulière comme une cristallisation de l'universel : Olympie, Homère.

\section{NOTES}

1. À supposer, toutefois, que les rites funéraires et les croyances relatives à l'au-delà fassent parti de la religion, du culte des dieux. Cela dépend du sens, large ou étroit, que l'on donne au mot de religion; cela dépend aussi des diverses religions : les croyances sur les dieux et celles sur l'au-delà peuvent former deux domaines séparés. Enfin, les rites funéraires ne supposent pas nécessairement des croyances sur l'au-delà : lorsque nousmêmes déposons des fleurs sur une tombe, nous ne supposons pas que le défunt viendra en humer le parfum.

\section{J.-C. Passeron, Le Raisonnement sociologique. L'espace non-popperien du raisonnement} naturel, Paris, Nathan, p. 391.

3. Dion de Pruse, XXVII, 5-6, qui attribue ce propos à Diogène ; même tripartition, attribuée à Pythagore, chez Cicéron, Tusculanes, V, 3, 9.

4. Philostrate, Vie d'Apollonios de Tyane, V, 34 ; Plutarque, Vie de Pélopidas, XXXIV, 6-7 ; Id., Ad Apollonium, 6 (Moralia, 105 b) ; Id., Non posse suaviter vivi, 19 (Moralia, $1100 \mathrm{c}$ ) ; Horace, Carmina, I, 1, 3 ; Philostrate, VI, 10 et V, 43 ; Aristote chez I. During, Der Protreptikos des Aristoteles, Francfort-sur-le-Main, Klostermann, 1969, p. 50.

5. Dion de Pruse, IX, 10-20.

6. Thucydide, VI, 16.

7. Dion de Pruse, IX, 21-22.

8. Dion de Pruse, Melancomas, XVIII, 7-8 et XXIX, 11.

9. Philostrate, Apollonios, 1,34 et IV, 28, cf. IV, 25.

10. L. Robert, Opuscula selecta, Amsterdam, Hakkert, 1989, vol. 5, p. 256.

11. Oracula Sibyllina, II, 39 (Kurfess, Sibyllinische Weissagungen, 1951, p. 54).

12. Plutarque, De fraterno amore, 5 (Moralia, $480 \mathrm{~b}$ ).

13. Inscriptiones Graecae, editio altéra, 11-111, 3155.

14. Philostrate, Apollonios, VI, 34. 
15. Pour tout ce qui suit, voir les travaux fondamentaux de H.W. Pleket, Zur Soziologie des antiken Sports, dans Mededelingen van het Nederlands Instituut te Rome, XXXVI, 1974 ; Games, prizes, athletes and ideology, dans Arena, 1, 1976; et d'Ingomar Weiler, Der Sport bei den Völkern der alten Welt, Darmstadt, Wiss. Buchgesellschaft, 1981. Citons aussi M. I. Finley et H. W. Pleket, The Olympic Games : the first thousand years, Londres, Chatto \&Windus, 1976 ; trad., Die Olympischen Spiele in der Antike, 1976.

16. Plutarque, Vie de Périclès, II, 1 ; Lucien, Somnium, 8-9 ; Sénèque, Ad Lucilium, Lettre 88, 2. 17. L. Robert dans les Entretiens sur l'antiquité classique (Vandœuvres-Genève, Fondation Hardt), XIV, 1969, p. 292.

18. H. W. Pleket dans Mededelingen, p. 57-59; dans Arena, op. cit., p. 53.

19. Pline L'Ancien, Histoire naturelle, XXXIV, 5; Pétrone, Satiricon, 88.

20. Vers la fin de Die Zeit des Weltbildes (dans les Holzwege), Heidegger écrit ainsi que « les Grecs n'ont jamais vécu leur fête olympique comme une expérience à avoir vécue ». Mais si, justement ; c'est là, de la part de Heidegger, une naïveté qui témoigne d'un manque de sens historique des réalités - et d'un sens politique, pour des époques plus récentes. Sur ce genre de problème, voir J.-P. Olivier de Sardan, "Occultism and the ethnographic "I". The exoticizing of magic from Durkheim to "postmodern" anthropology ", Critique of Anthropology, 12 (1), 1992. p. 5-25.

21. I. Weiler, Der Sport bei den Vôlkern der alten Welt, p. 53 et 83.

22. Sénèque, Ad Lucilium, lettre 76, 4 (Seneca, Philosophische Schriften, trad. M. Rosenbach, Darmstadt, Wiss. Buchgesellschaft, 1987, vol. 5, p. 97).

23. Nous poussons l'intrépidité jusqu'à supposer que les gens peuvent s'intéresser réellement au sport ou à la musique et qu'il ne faut pas ramener leurs objets à des symboles qui ne serviraient qu'à manifester, soit une « identité » nationale, soit de la « distinction » sociale.

24. On retrouve ce fonctionnalisme dans la sociologie de la « distinction » de P. Bourdieu, qui semble réduire l'esthétique à une recherche de la différence valorisante par une classe sociale.

25. H. W. Pleket dans Mededelingen, p. 73.

26. L. Robert, dans les Praktika du VIII ${ }^{\mathrm{e}}$ congrès d'épigraphie grecque et latine, Athènes, 1984, vol. I., p. 45.

27. Depuis 208 avant notre ère (Livius, XXVII, 35, 3).

28. Mastanabal, fils d'un roi berbère hellénisé et hellénisateur, fut admis aux Panathénées et son char y fut vainqueur (Inscriptiones Grœcœ, II-III, editio minor, 2316, 41).

29. Le futur empereur Tibère (W. Dittenberger, Die Inschriften von Olympia, 16) ; J. H. Krause, Olympia oder Darstellung der grossen olympischen Spiele (1838 ; réimp. G. Olms, Hildesheim, 1972), p. 48, n. 33-34.

30. Lucien, De morte Peregrini, 35.

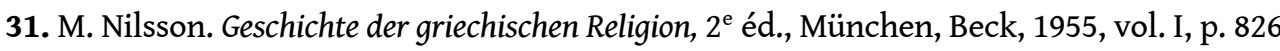
sq.

32. J. Piaget, La formation du symbole chez l'enfant, Neuchatel, 1945, p. 164.

33. M. I. Finley et H. W. Pleket, The Olympic Games, p. 22. Ajoutons que, jusqu'au cinquième siècle, les athlètes qui venaient concourrir à Olympie étaient surtout des Spartiates et que le Péloponnèse était probablement une terre de champions.

34. J.-C. Passeron, Le Raisonnement sociologique..., p. 324-333.

35. Pindare, Première Olympique.

36. G. Simmel, Philosophische Kultur, Berlin, Wagenbach, 1983, p. 183. 